\title{
Generation of Organotypic Multicellular Spheres by Magnetic Levitation: Model for the Study of Human Hematopoietic Stem Cells Microenvironment
}

\author{
Claudia Camila Mejía-Cruz ${ }^{1}$, Emilia Barreto-Durán ${ }^{1}$, María Alejandra Pardo-Pérez ${ }^{1}$,
} María Camila Jimenez ${ }^{1}$, Julieth Rincón ${ }^{1}$, Karen Vanegas ${ }^{1}$, Jorge Luis Rodríguez ${ }^{2}$, Luis Fernando Jaramillo-Garcia ${ }^{2}$, Juan Carlos Ulloa ${ }^{3}$, Rodolfo Martínez Díaz ${ }^{4}$ Efrain Leal-García, Rafael Pérez-Núñez, Alfonso Barreto ${ }^{1}$, Viviana M Rodríguez-Pardo ${ }^{1}$

${ }^{1}$ Immunobiology and Cell Biology Group, Department of Microbiology, Science Faculty, Pontificia Universidad Faveriana, Bogotá D.C., Colombia, ${ }^{2}$ Department of Pathology, School of Medicine, Pontificia Universidad Faveriana, Hospital Universitario San Ignacio, Bogotá D.C., Colombia, ${ }^{3}$ Virology Group, Department of Microbiology, Science Faculty, Pontificia Universidad Faveriana, Bogotá D.C., Colombia, ${ }^{4}$ Department of Gynecology and Obstetrics, School of Medicine, Pontificia Universidad Faveriana, Hospital Universitario San Ignacio, Bogotá D.C., Colombia, ${ }^{5}$ Department of Orthopedics and Traumatology, School of Medicine, Pontificia Universidad Javeriana, Hospital Universitario San Ignacio, Bogotá D.C., Colombia

Background and Objective: The characteristics of human hematopoietic stem cells are conditioned by the microenvironment of the bone marrow, where they interact with other cell populations, such as mesenchymal stem cells and endothelial cells; however, the study of this microenvironment is complex. The objective of this work was to develop a 3D culture system by magnetic levitation that imitates the microenvironment of human HSC.

Methods and Results: Human bone marrow-mesenchymal stem cells, umbilical cord blood-hematopoietic stem cells and a non-tumoral endothelial cell line (CC2811, Lonza ${ }^{\mathbb{R}}$ ) were used to develop organotypic multicellular spheres by the magnetic levitation method. We obtained viable structures with an average sphericity index greater than 0.6 , an average volume of $0.5 \mathrm{~mm}^{3}$ and a percentage of aggregation greater than $70 \%$. Histological studies of the organotypic multicellular spheres used hematoxylin and eosin stains, and an evaluation of vimentin expression by means of immunohistochemistry demonstrated an organized internal structure without picnotic cells and a high expression of vimentin. The functional capacity of human hematopoietic stem cells after organotypic multicellular spheres culture was evaluated by multipotency tests, and it was demonstrated that 3D structures without exogenous Flt3L are autonomous in the maintenance of multipotency of human hematopoietic stem cells.

Conclusions: We developed organotypic multicellular spheres from normal human cells that mimic the microenvironment of the human hematopoietic stem cells. These structures are the prototype for the development of complex organoids that allow the further study of the biology of normal human stem cells and their potential in regenerative medicine.

Keywords: Organotypic multicellular spheres, Organoids, Hematopoietic stem cells, Mesenchymal stem cells, Microenvironment

Received: August 2, 2018, Revised: October 24, 2018, Accepted: November 29, 2018, Published online: February 28, 2019

Correspondence to Viviana M Rodríguez-Pardo

Department of Microbiology, Science Faculty, Javeriana University, Carrera 7a No 43-82. Edificio Carlos Ortiz (Ed. 52), Bogotá D.C. 110231, Colombia

Tel: +571-3208320-4072, Fax: +571-3208320-4021, E-mail: vivianar@javeriana.edu.co

(a) This is an open-access article distributed under the terms of the Creative Commons Attribution Non-Commercial License (http://creativecommons.org/licenses/by-nc/4.0/), which permits unrestricted non-commercial use, distribution, and reproduction in any medium, provided the original work is properly cited.

Copyright (c) 2019 by the Korean Society for Stem Cell Research 


\section{Introduction}

Since Schofield presented the "niche concept" in the 1970s (1), it has been recognized that the bone marrow (BM) microenvironment is important in controlling the biological characteristics of hematopoietic stem cells (HSC) with the support of cell populations such as osteoblasts, neurons, mesenchymal stem cells (MSC) and endothelial cells $(\mathrm{Ec})$, among other cells $(2,3)$. Several studies have shown that these interactions between HSC and other cellular populations in the BM are organized in two main complex niches: endosteal, close to the bone and influenced by osteoblasts, osteocytes, osteoclasts and vascular formed by sinusoids, arterioles and different populations of mesenchymal cells (stem cells and progenitors of bone lineage, cartilage and adipose tissue) $(3,4)$. In particular, the vascular niche is constituted of Ec, which forms a network of sinusoids and arterioles to which perivascular stromal cells from the heterogeneous group of BM-MSC are associated $(5,6)$. Ec participate in the homeostasis of hematopoiesis by secreting angiogenic factors that stimulate the proliferation and self-renewal of HSC by increasing the expression of factors such as IGFBP2, FGF2, BMP4 and DHH (7) and direct contact through E-selectin that promotes the quiescence of hematopoietic cells (8); furthermore, Ec has been shown to promote the long-term maintenance of HSC in vitro $(9,10)$.

Similarly, BM-MSC have an important role in the regulation of HSC. These cells, which express common mesenchymal antigens such as CD73 and CD105 or particular antigens (according to the subtype) such as CD146, CXCL-12 (CAR cells), nestin and leptin receptor (LepR), have in common the secretion of two determining soluble factors for HSC: CXCL-12 (SDF-1) and SCF (11, 12), which participate in maintaining the viability, self-renewal and mobilization of hematopoietic cells. Some MSC populations of the vascular niche are more resistant to chemotherapy and induce the regeneration of HSC after exposure to cytotoxic agents (13), and in vitro, they can maintain the phenotypic and functional characteristics of HSC of umbilical cord blood and leukemic cells $(14,15)$.

These findings demonstrate that the Ec and MSC of the microenvironment $\mathrm{BM}$ have a determining role in the regulation of the mechanisms that maintain the homeostasis of the HSC; however, to deepen our understanding of role of the microenvironment in the regenerative capacity of HSC and the knowledge about the maintenance of cancer stem cells and thus be able to propose therapeutic strategies, it is necessary to develop experimental systems that imitate the microenvironment of human HSC with the si- multaneous use of the different cell populations that reside in the niches of the hematopoietic cells. The use of animal models has allowed significant advancements in the knowledge of the microenvironment of HSC (16-18); however, studies show that murine HSC and human HSC interact differently with cells in the bone marrow microenvironment (19) and that species-specific differences may exist (20). An interesting alternative for the study of the microenvironment of human HSC is the development of organotypic multicellular spheres (OMS) that mimic the niches of human cells (21). In this work, we propose a new system of 3D culture by magnetic levitation, which does not use scaffolds or exogenous matrices and allows the cells to form their own extracellular matrix, to evaluate the role of Ec and MSC in the behavior of the human HSC as a future tool for the development of a complex organoid that imitates the microenvironment of the human BM.

\section{Materials and Methods}

Isolation and culture conditions of human MSC and Ec

To obtain MSC, bone marrow (BM) samples were obtained with the support of the Department of Orthopedics and Traumatology of Hospital Universitario San Ignacio (Bogota, Colombia) from volunteer donors (undergoing hip replacement surgery) after signing the informed consent form approved by the Hospital Ethics Committee. BM-MSC were isolated and cultured as previously published $(14,15)$, and they were used in passages $3-5$ for a 3D culture system. The BM-MSC phenotype was assessed by flow cytometry with a FACS Aria-II cytometer (BD Biosciences). For data analysis, the DIVA (BD Biosciences) and FlowJo software were used. A BM-MSC functional assay was performed according to previous reports $(14,15)$ with StemPro Osteogenesis, StemPro Adipogenesis and StemPro Chondrogenesis Differentiation Kits (Invitrogen). A non-tumor cell line, Human Dermal Microvascular Endothelial Cells (CC-2811, Lonza), was used. The endothelial cells were cultured in an EGM-2 Bullet medium kit (Lonza) at a density of $5 \times 10^{3} / \mathrm{cm}^{2}$ at $37^{\circ} \mathrm{C}$ and $5 \% \mathrm{CO}_{2}$. The viability of the cell populations was monitored permanently with trypan blue staining.

\section{Isolation and characterization of HSC from human umbilical cord blood (UCB)}

To obtain HSC, umbilical cord blood (UCB) samples were obtained with the support of the Department of Gynecology and Obstetrics of Hospital Universitario San Ignacio (Bogota, Colombia) from normal full-term deliv- 
eries after obtaining informed consent in accordance with the guidelines approved by the Hospital Ethics Committee. Mononuclear cells were isolated by Ficoll density gradient centrifugation (Histopaque $\mathrm{d}=1.077 \mathrm{~g} / \mathrm{cm}^{3}$, Sigma-Aldrich), and CD34+ cells were isolated using a previously described magnetic-activated cell-sorting (MACS) CD34 isolation kit (Miltenyi Biotec) (15). Cell purity was evaluated by flow cytometry, and cell viability was determined using trypan blue staining.

\section{Generation of the 3D cultivation system by magnetic levitation}

To develop a $3 \mathrm{D}$ culture system that mimics the microenvironment of the bone marrow, a 3D Bio Assembler ${ }^{\mathrm{TM}}$ System (Nano3D Biosciences, Inc.) was used with the three cell populations of interest (MSC, Ec and HSC). It was formed of a 24-Well Bio-Assembler ${ }^{\mathrm{TM}}$ Kit with nanoparticles (NanoShuttleTM-PL) coated with iron oxide (Fe2O3), gold and poly-lysine-L, magnetic drag plates (24-Well Magnetic Drive ${ }^{\circledR}$ ) and ultra-low adhesion plates (Ultra Low Adhesion 24-Well Plates ${ }^{\circledR}$ ). Each of the isolated and characterized cell populations (MSC, Ec and HSC) was independently cultured with $400 \mu 1$ of triple medium formed in equal parts of serum-free medium for MSC (StemPro MSC SFM CTS ${ }^{\mathrm{TM}}$, GIBCO, Life Technologies), HSC (StemPro34 SFM, GIBCO, Life Technologies) and Ec (Endothelial Cell Growth Medium, EGM ${ }^{\mathrm{TM}}-2$ BulletKit ${ }^{\mathrm{TM}}$ Lonza), with a ratio of $1 \mu 1$ of nanoparticles per $1 \times 10^{4}$ cells (22) for 12 hours at $37^{\circ} \mathrm{C}$ and $5 \% \mathrm{CO}_{2}$. Then, all cells were collected and mixed in the ultra-low adhesion plate (Ultra Low Adhesion 24-Well Plates ${ }^{\circledR}$ ) with $400 \mu 1$ of triple medium, and a magnetic drag plate (24Well Magnetic Drive $\left.{ }^{(}\right)$was assembled and incubated for 120 hours at $37^{\circ} \mathrm{C}$ and $5 \% \mathrm{CO}_{2}$. To define the optimal relationship between the volume of nanoparticles and cell density in the 3D culture system, the morphology of the structure obtained (organotypic multicellular spheres, OMS) was evaluated by inverted microscopy, and the global DNA content was determined with a High Pure PCR Template Preparation Kit (Roche) and NanoDrop 2000 (Thermo Scientific) in two different cell-to-cell densities, MSC: Ec: $H S C=1: 5: 5$ and 1:2:2 with $1 \times 10^{5}$ total cell density per well.

\section{Evaluation of OMS: volume, sphericity, aggregation and viability}

To calculate the volume and sphericity of the structures obtained by the 3D culture method by magnetic levitation, a photographic record of the spheres was made during days 5,10 and 15 of culture; then, with the Image $J^{\circledR}$ soft- ware, the data of area (A), perimeter (P) and diameter (D) were obtained. The volume $(\mathrm{V})$ was determined according to $\mathrm{V}=4 \pi / 3(\mathrm{P} / \pi)^{3}(23)$, and the sphericity $(\Phi)$ was determined with the calculation $\Phi=\pi(\sqrt{4 \mathrm{~A} /} \pi) / \mathrm{P}$ (23). The percentage of aggregation (\%Ag) in the sphere was calculated to determine the loss of cells during the formation of the structure (5 day), it was determined according to Martin et al. (24) (\% $\mathrm{Ag}=100$ (TC-SC)/TC; TC: total number of plated cells, SC: number of single cells counted after filtering. To determine the viability of the OMS, a LIVE/DEAD ${ }^{\mathrm{TM}}$ Cell Imaging Kit (Invitrogen) was used following the manufacturer's instructions. 3D structures were imaged with laser scanning confocal microscope FV1000 (Olympus) using an UPLSAPO 20X (1.35 NA oil immersion objective), $488 \mathrm{~nm}$ argon laser line for green fluorescence (living cells) and HeNe $543 \mathrm{~nm}$ laser for red fluorescence (dead cells). Data analysis was performed with the FlowView and Fiji software. Previous studies have shown that the addition of cytokines during the in vitro expansion of HSC favors their viability and proliferation $(25,26)$. To determine whether the OMS obtained were autonomous in the maintenance of long-term HSC (10 and 15 days of culture) or required exogenous cytokines, an addition of Flt3-L (100 ng/ml) was used (27).

\section{Histochemical characteristics of spheres}

To evaluate the internal structure of the OMS, they were manipulated with the MagPen ${ }^{\mathrm{TM}}$ (Nano3D Biosciences, Inc.) and embedded in paraffin. Then, 3- $\mu \mathrm{m}$-thick sections were cute, deparaffinized and stained in the Pathology Department of San Ignacio University Hospital (Bogotá, Colombia). The sections were stained with hematoxylin-eosin. The expression of vimentin (clone V9, IgG1. Agilent ${ }^{\circledR}$ ) was determined by immunohistochemistry and Ki67 antigen was detected by confocal microscopy (clone

Table 1. Bone marrow donors for MSC in vitro expansion

\begin{tabular}{ccccc}
\hline No & Age (years) & $\begin{array}{c}\text { Gender } \\
\text { (Female } \\
\text { /Male) }\end{array}$ & $\begin{array}{c}\text { MNC } \\
\text { total counts }\end{array}$ & $\begin{array}{c}\text { Volume } \\
\text { BM (ml) }\end{array}$ \\
\hline 1 & 31 & F & $71 \times 10^{6}$ & 45 \\
2 & 41 & F & $46.5 \times 10^{6}$ & 35 \\
3 & 64 & F & $10.5 \times 10^{6}$ & 25 \\
4 & 56 & M & $15.2 \times 10^{6}$ & 20 \\
5 & 63 & F & $41.5 \times 10^{6}$ & 15 \\
6 & 27 & F & $116 \times 10^{6}$ & 27 \\
7 & 40 & F & $48 \times 10^{6}$ & 40 \\
8 & 20 & F & $45 \times 10^{6}$ & 20 \\
\hline
\end{tabular}




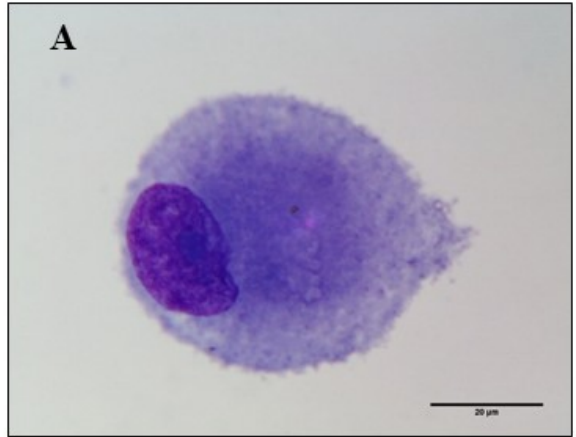

C

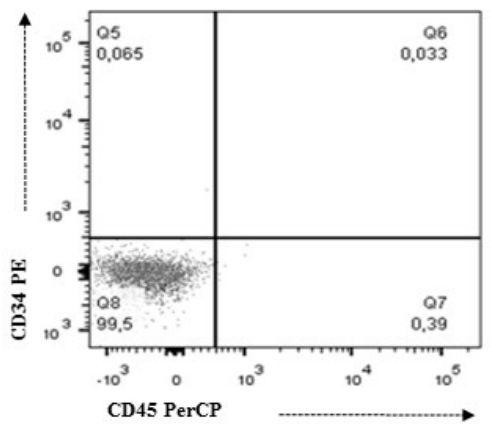

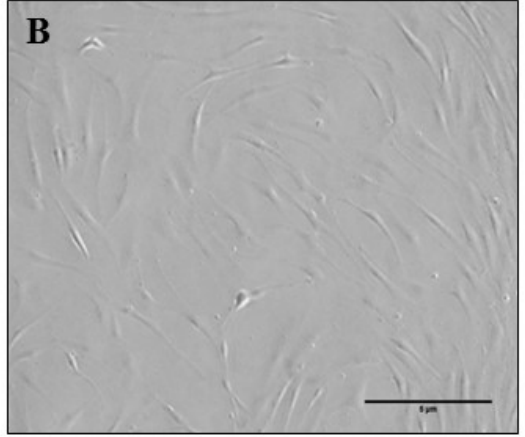

$\mathbf{D}$

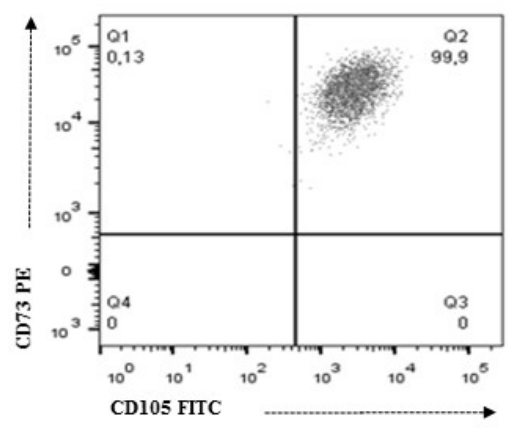

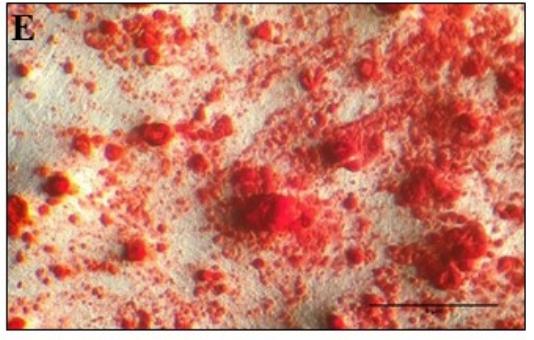
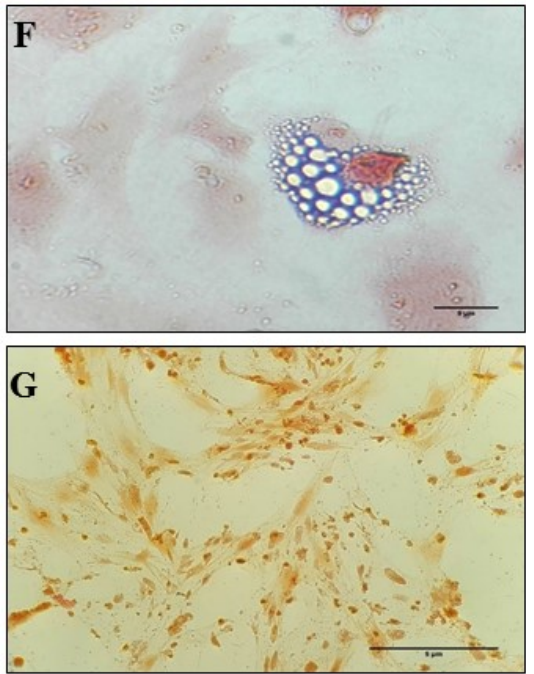

Fig. 1. MSC characteristics isolated from human BM. (A) MSC Morphology (3 passage): cells with an eccentric nucleus, loose chromatin and nucleoli; presence of granular basophilic cytoplasm ( $\times 100$ Cythospin, hematoxylin and eosin stain, optical microscopy. Scale bar: $20 \mu \mathrm{m})$, (B) MSC Morphology (3 passage): Fibroblastoid cells associated with MSC ( $\times 20$, inverted microscopy. Scale bar: $5 \mu \mathrm{m}),(\mathrm{C}$, D) Immunophenotype (3 passage): MSCs do not express hematopoietic antigens (CD34 and CD45) and co-express CD73 and CD105, (E) Osteogenic differentiation $(\times 20$, red alizarin stain, inverted microscopy. Scale bar: $5 \mu \mathrm{m})$, (F) Adipogenic differentiation $(\times 40$, sudan black stain, inverted microscopy. Scale bar: $5 \mu \mathrm{m}),(\mathrm{G})$ Chondrogenic differentiation $(\times 20$, safranin $\mathrm{O}$ stain, inverted microscopy. Scale bar: $5 \mu \mathrm{m})$.

Table 2. Percentage of antigens evaluated in mesenchymal cells isolated from bone marrow

\begin{tabular}{ccccc}
\hline No & CD105 & CD73 & CD34 & CD45 \\
\hline 1 & $98.2 \%$ & $100 \%$ & $0.097 \%$ & $0 \%$ \\
2 & $99 \%$ & $100 \%$ & $0.11 \%$ & $0.25 \%$ \\
3 & $99.8 \%$ & $100 \%$ & $0.14 \%$ & $1.13 \%$ \\
4 & $99.9 \%$ & $100 \%$ & $0.27 \%$ & $0.34 \%$ \\
5 & $98.5 \%$ & $99.6 \%$ & $0.68 \%$ & $0.95 \%$ \\
6 & $99.6 \%$ & $99.8 \%$ & $0.43 \%$ & $1.56 \%$ \\
7 & $100 \%$ & $99.4 \%$ & $3.68 \%$ & $0.11 \%$ \\
8 & $100 \%$ & $100 \%$ & $0.57 \%$ & $0 \%$ \\
\hline
\end{tabular}

4A1, Thermo Scientific ${ }^{\circledR}$, dilution $\left.1 / 200\right)$. To determine the percentage of expression of Ki67, the total area of the sphere was determined according to the expression of $\alpha$ tubulin and the expression area of Ki67 with the use of Image ${ }^{\mathrm{R}}$ software, then the area of expression of the antigen (Ki67) was calculated with the following formula: $\operatorname{Ki67}(\%)=\operatorname{Ki67}$ area $(\mu \mathrm{m})^{*} 100 / \alpha$-tubulin area $(\mu \mathrm{m})$.
Table 3. Umbilical cord blood (UCB) donors for HSC isolation

\begin{tabular}{cccccc}
\hline NO & $\begin{array}{c}\text { Maternal } \\
\text { donor age } \\
\text { (years) }\end{array}$ & $\begin{array}{c}\text { Gestation } \\
\text { weeks }\end{array}$ & $\begin{array}{c}\text { Volume } \\
\text { UCB } \\
(\mathrm{ml})\end{array}$ & $\begin{array}{c}\text { CD34+total } \\
\text { counts }\end{array}$ & $\begin{array}{c}\text { CD34+ } \\
\text { (\% purity) }\end{array}$ \\
\hline 1 & 26 & 40 & 41 & $170 \times 10^{3}$ & 90.45 \\
2 & 27 & 38 & 61 & $130 \times 10^{3}$ & 97.20 \\
3 & 29 & 37 & 48 & $260 \times 10^{3}$ & 95.32 \\
4 & 26 & 36 & 60 & $250 \times 10^{3}$ & 91.42 \\
5 & 32 & 37 & 50 & $80 \times 10^{3}$ & 90.34 \\
6 & 18 & 37 & 55 & $940 \times 10^{3}$ & 94.34 \\
7 & 31 & 39 & 16 & $1400 \times 10^{3}$ & 82.4 \\
8 & 38 & 37 & 31 & $400 \times 10^{3}$ & 85.7 \\
9 & 21 & 38 & 40 & $1000 \times 10^{3}$ & 96.3 \\
10 & 26 & 38 & 36 & $1800 \times 10^{3}$ & 89.7 \\
11 & 24 & 40 & 24 & $2000 \times 10^{3}$ & 97 \\
\hline
\end{tabular}

\section{Functional evaluation of HSC}

To evaluate the maintenance of the biological characteristics of the HSC cultured in the OMS, the multipotent capacity of HSC was evaluated after 10 and 15 days of the 
3D culture. To isolate the HSC from the OMS, an enzymatic treatment with 1X trypsin and EDTA (Gibco Life Technologies) was performed for 30 minutes at $37^{\circ} \mathrm{C}$. Subsequently, HSC were sorted by a negative selection using anti-CD105 antibody (antigen expressed by MSC and Ec) and LS columns (magnetic-activated cell-sorting, MACS. Miltenyi Biotec). From the collected HSC, a new determination was made by flow cytometry to evaluate the purity of the recovered hematopoietic cell population, and a multipotency test was performed with the use of the HSC-CFU complete with EPO medium (Miltenyi Biotec ${ }^{\circledR}$ ) during 15 days at $37^{\circ} \mathrm{C}$ and $5 \% \mathrm{CO}_{2}$.

\section{Statistical analysis}

Shapiro-Wilk test was applied to contrast the normality of the data. Mann-Whitney U test was used for the comparison of means and Tukey test for multiple comparisons. A 95\% confidence interval was defined to accept a difference as significant when $\mathrm{p}$ less was than $0.05(\mathrm{p}<0.05)$ (Software Graph PadPrism6).
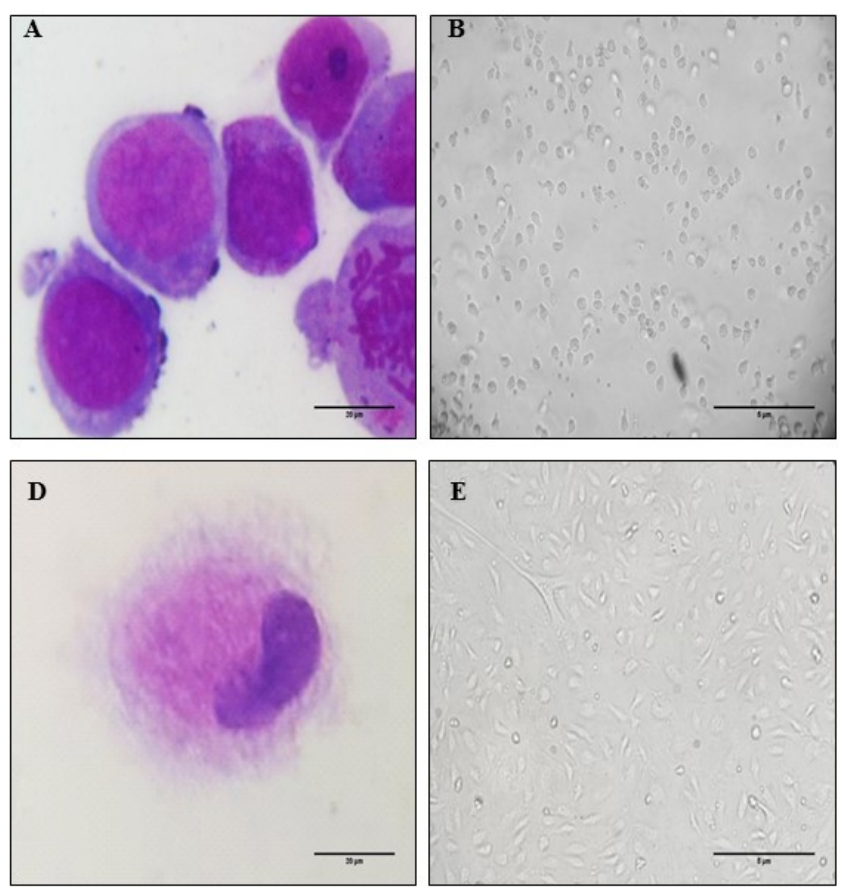

\section{Results}

\section{Isolation, culture and characterization of cell populations: MSC, Ec and HSC}

To collect and isolate MSC, eight BM samples were collected from voluntary donors (Table 1). According to the ISCT recommendations (28), MSCs isolated from all the samples showed the morphological characteristics, immunophenotype and differentiation capacity (osteogenesis, chondrogenesis and adipogenesis) of this cell population (Fig. 1, Table 2). Eleven UCB samples were collected to isolate CD34+HSC with a percentage of isolation greater than $80 \%$ (Table 3). The morphological and immunophenotypic characteristics of UCB-HSC and endothelial cells (Ec) are presented in Fig. 2.

\section{Relationship between cell populations and nanoparticles to obtain OMS}

To generate stable OMS, the optimal relationship between the volume of nanoparticles and the density of each of the cell populations (MSC, Ec and HSC) was defined by evaluating the morphology and DNA content in each of the structures after 5 days. Fig. 3 shows the formation

$\mathrm{C}$
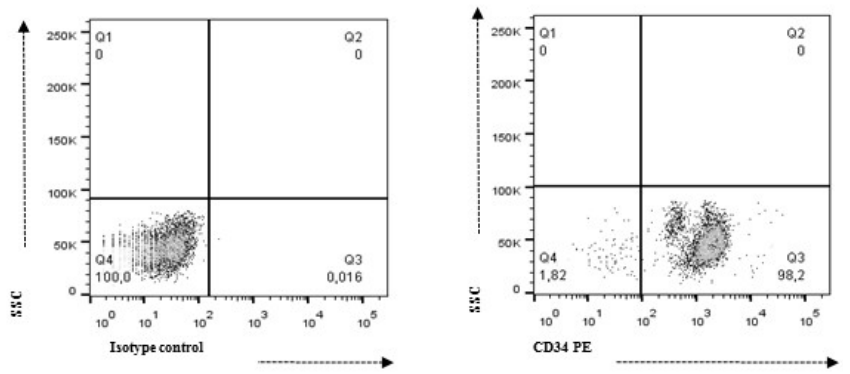

$\mathbf{F}$
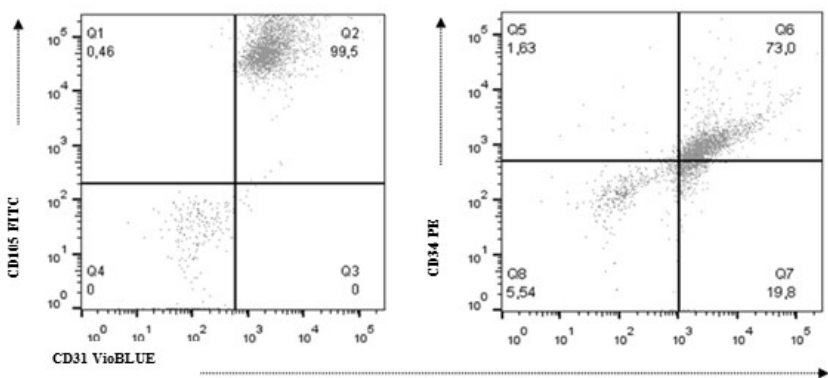

Fig. 2. UCB-HSC and Ec characteristics (cell line CC-2811. Lonza ${ }^{\circledR}$ ). (A) HSC Morphology: cells with a predominant nucleus, nucleoli and basophil cytoplasm ( $\times 100$ Cythospin, hematoxylin and eosin stain, optical microscopy. Scale bar: $20 \mu \mathrm{m}),(B)$ Non-adherent cells compatible with UCB-HSC $(\times 20$, inverted microscopy. Scale bar: $5 \mu \mathrm{m}),(\mathrm{C})$ HSC phenotype: CD34+, (D) Ec Morphology: cells with metacentric nucleus and abundant cytoplasm $(\times 100$ Cythospin, hematoxylin and eosin stain, optical microscopy. Scale bar: $20 \mu \mathrm{m}),(\mathrm{E})$ Adherent cells compatible with Ec $(\times 20$, inverted microscopy. Scale bar: $5 \mu \mathrm{m}),(\mathrm{F})$ Ec phenotype: CD34+, CD31+, CD105+. 

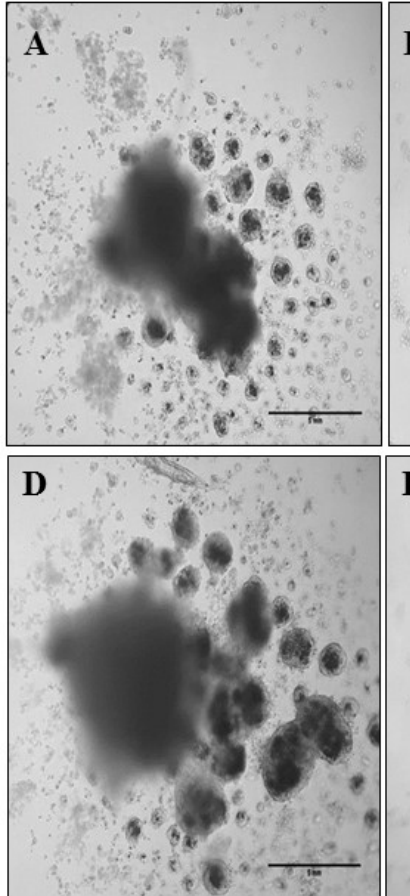

96h
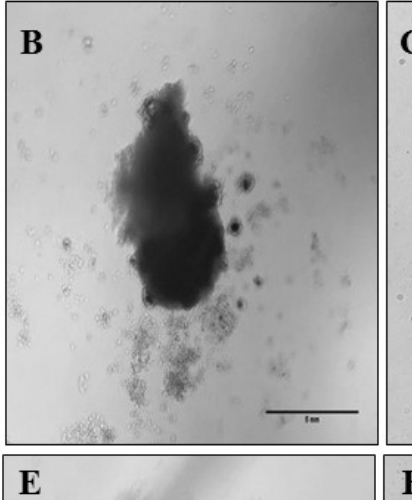

E

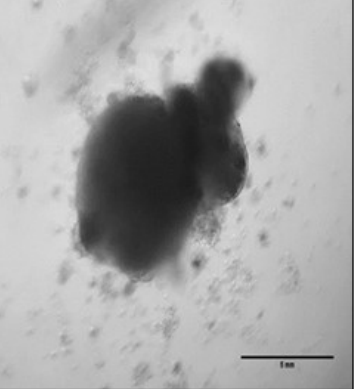

$120 \mathrm{~h}$

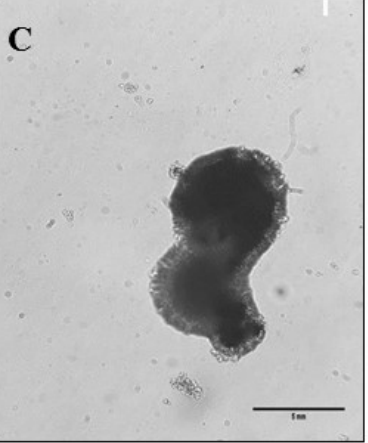

F
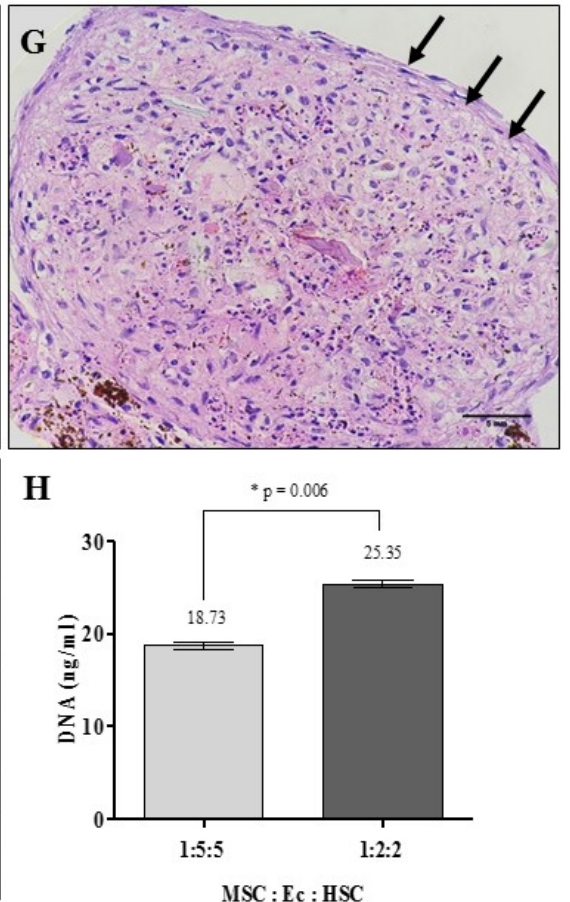

\section{.}

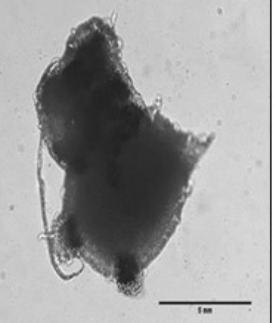

Fig. 3. Organotypic multicellular spheroid (OMS) formation after 5 days: time vs. cell density. (A C Relation 1:5:5 MSC:EC:HSC ( $\times 20$, inverted microscopy. Scale bar: $5 \mathrm{~mm})$, (D F) Relation 1:2:2 MSC:Ec:HSC (Total cell density $1 \times 10^{5}$ cells) $(\times 20$, inverted microscopy. Scale bar: $5 \mathrm{~mm}$ ). (G) Morphology of OMS after 5 days of culture. It shows cells that are organized in the periphery of the structure demilitarizing the sphere (Relation 1:2:2 MSC:Ec:HSC. $\times 40$, hematoxylin \& eosin. Scale bar: $5 \mathrm{~mm}$ ). (H) DNA quantification in the spheres of the two experimental conditions $(p<0.05)$.

5 d

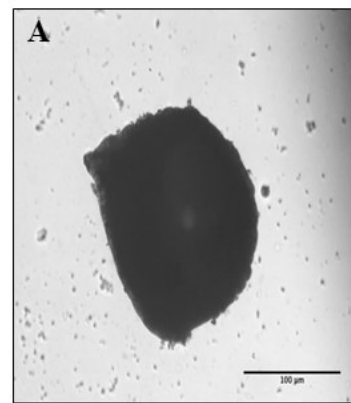

D

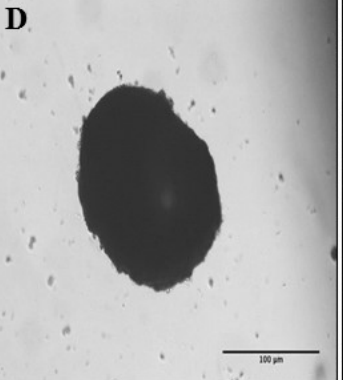

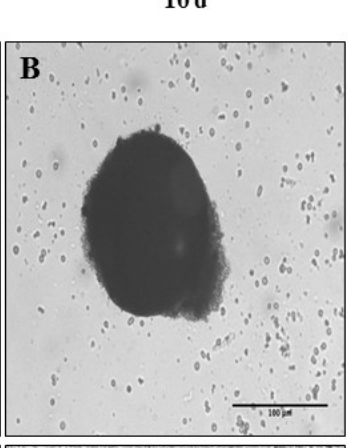

E

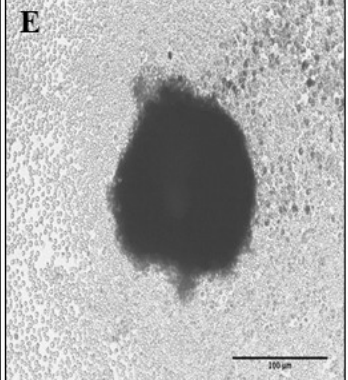

$15 d$
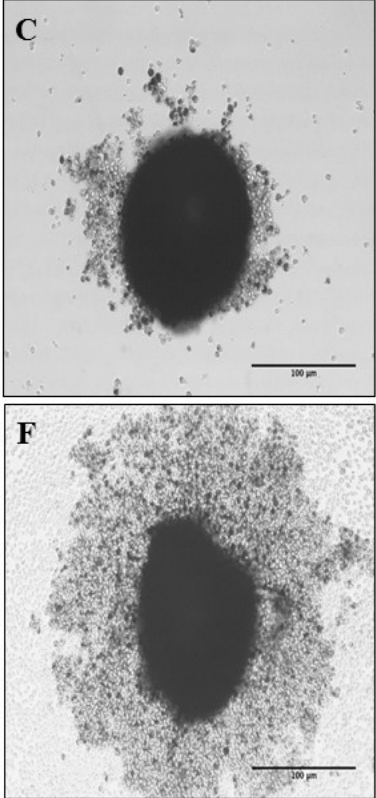
of the OMS according to the different cell-to-cell densities and DNA content for experimental conditions. Fig. 3G shows the internal structure of the OMS after 5 days of cultivation, and we observed that some cells are organized in the periphery of the structure "limiting" the sphere in an orderly manner. According to the morphological characteristics and DNA content of the OMS evaluated, MSC: Ec: HSC cell ratio of 1:2:2 was selected to evaluate the efficiency of the 3D culture system by magnetic levitation to mimic the human $\mathrm{BM}$ microenvironment.

\section{General characteristics of multicellular spheres}

The OMS (MSC: Ec: HSC of 1:2:2) was evaluated after 5,10 and 15 days with and without the exogenous addition of $100 \mathrm{ng} / \mathrm{ml}$ of Flt3L to determine the autonomy of structures in long-term HSC maintenance. Fig. 4 shows OMS without and with the exogenous addition of the cytokine. Fig. 4F shows an OMS with peripheral halo possibly associated with cells in the proliferation state (15 days). It was demonstrated that there were no significant differences in the percentage of aggregation between the spheres with and without the exogenous addition of cytokines (Fig. 4G, $82.35 \%$ vs. $71.41 \%$ ), showing that in this $3 \mathrm{D}$ culture system, there was only a loss of $30 \%$ of the cells with which the culture was started. Similarly, no significant differences were observed in the sphericity and volume of the spheres evaluated; it is still important to indicate that during the days of cultivation, while the sphericity increases the volume decreases (Fig. 5A, B). Interestingly, we observed that after 10 and 15 days, the OMS are structures that conserve a high cellular viability (Fig. 5C-F).

\section{Histological evaluation of the spheres}

To evaluate the internal structure of the OMS (without and with Flt3L), a morphological assessment was made after 15 days of culture. Fig. 5G, H shows the structures of the OMS with hematoxylin and eosin staining, demonstrating that in the center of the spheres, no cells with pyknotic nuclei associated with cell death were observed. In
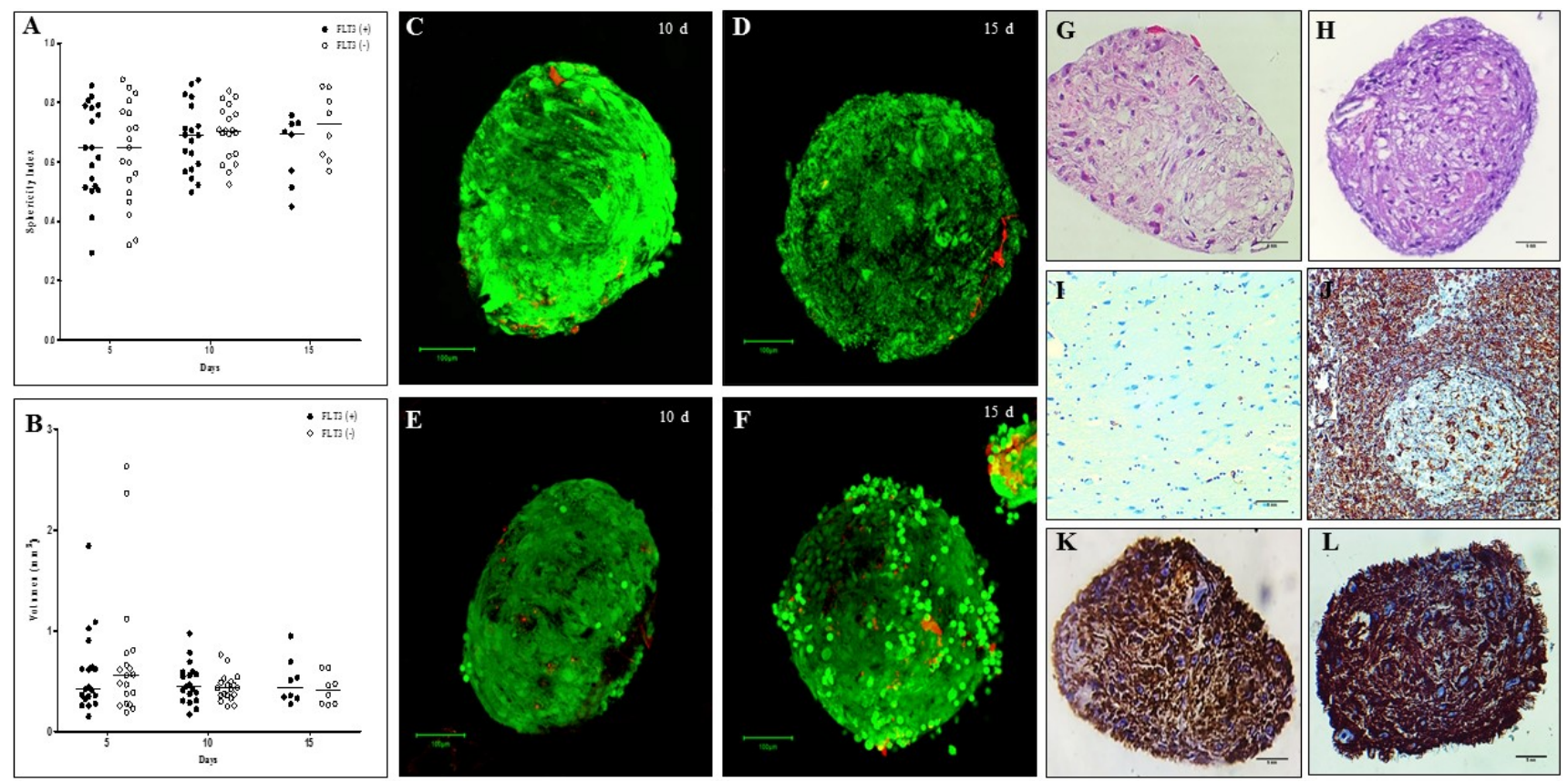

Fig. 5. Sphericity, volume, viability and histological evaluation of organotypic multicellular spheroid (OMS). (A) Sphericity Index, non significant differences were observed between the spheres cultivated without and with Flt3L, (B) Volumen, similarly there are no differences between the experimental conditions $(\mathrm{p}>0.05)$. 3D structures were treated with exogenous Flt3L (100 ng/ml) $(C$, D) or without cytokine (E-F). Viability was evaluated with LIVE/DEAD ${ }^{\mathrm{TM}}$ Cell Imaging Kit (Invitrogen) and confocal microscope FV1000 (Olympus) using an UPLSAPO $20 \times(1.35 \mathrm{NA}$ oil immersion objective), $488 \mathrm{~nm}$ argon laser line for green fluorescence (living cells) and HeNe $543 \mathrm{~nm}$ laser for red fluorescence (dead cells). Data analysis was performed with the FlowView and Fiji software (green cells: alive, red cells: dead) (Scale bar: $100 \mathrm{um}$ ). For histological evaluation, after 15 days of culture, cells without pyknotic nuclei are observed in the OMS (G) without Flt3L $(\times 40$, hematoxylin \& eosin. Scale bar: $5 \mathrm{~mm})$ and $(\mathrm{H})$ with Flt3L $(\times 40$, hematoxylin \& eosin. Scale bar: $5 \mathrm{~mm})(\mathrm{I}) \mathrm{Negative} \mathrm{control}$ of vimentin (brain tissue) $(\times 40$. Scale bar: $5 \mathrm{~mm})$, (J) Positive control of vimentin (lymph node) $(\times 40$. Scale bar: $5 \mathrm{~mm})$, (K) Vimentina in OMS without Flt3L $(\times 40$. Scale bar: $5 \mathrm{~mm})$ and $(\mathrm{L})$ OMS with Flt3L $(\times 40$. Scale bar: $5 \mathrm{~mm})$. 
addition, it was shown that the structures obtained have an "organized morphology". Compared to the controls, the OMS shows a strong expression of vimentin (Fig. $5 \mathrm{~K}, \mathrm{~L}$ ). The expression of Ki67 was evaluated after 10 and 15 days and a greater tendency of expression was observed in the sphere with Flt3L, although the difference was not significant (Fig. 6).

\section{Functional evaluation of HSC after cultivation in multicellular spheres}

To establish whether the spheres obtained can mimic the microenvironment of the bone marrow, it is essential to evaluate the function of the HSC after a long period of culture within the spheres. To determine this, HSC multipotency tests were performed to evaluate the ability of these cells to generate CFU-GEMM (multipotent progenitors), CFU-GM (granulocyte-monocyte progenitors) and CFU-E (erythroid progenitors) after 10 and 15 days in culture inside the OMS and we observed that in comparison to CFU obtained from freshly purified HSC (basal) and HSC cultivated in the spheres with cytokine, the cultured HSC in the OMS without cytokine have a great capacity to generate more primitive hematopoietic colonies (CFU-GEMM) (Fig. 7).

\section{Discussion}

From the work of Moscona and Moscona (29), during recent years, it has been demonstrated that the organotypic multicellular spheres (OMS) developed in vitro can exhibit physiological characteristics similar to tissues in vivo, such as myocardium, hepatic and vascular tissue (30, 31). The formation of OMS involves the participation of different adhesion molecules, such as E-cadherin, N-cadherin, connexins and pannexins, as well as extracellular matrix (ECM) proteins, such as type I collagen and the activation of cytoskeletal proteins as actin filaments (32, 33 , which generates in the cells morphological changes that promote their aggregation and compaction with a decrease in their volume compared to the initial phase (34, 35). The OMS developed in our work have a similar behavior. We observed cellular aggregates that progressively compact in a structure with a sphericity that increases, while the volume decreases after 15 days of culture (Fig. 5A, B), which could demonstrate that in our system, there is possibly a dynamic between different adhesion molecules, ECM and structural changes in the cells.

It is important to consider that several models for the formation of OMS use cells isolated from solid tumors (for example, mammospheres), which spontaneously form

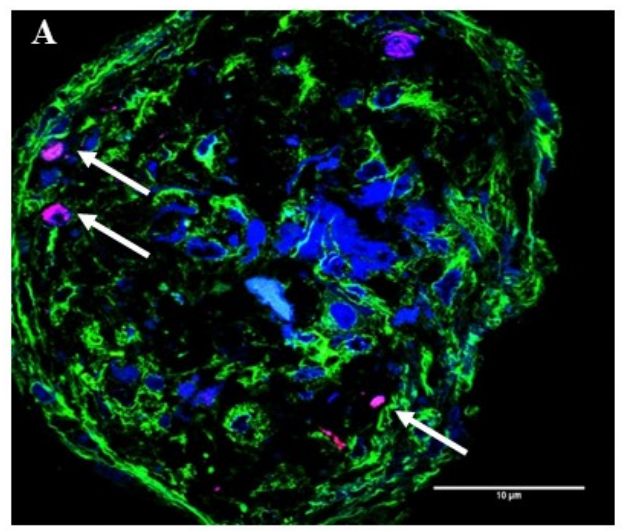

\section{B}
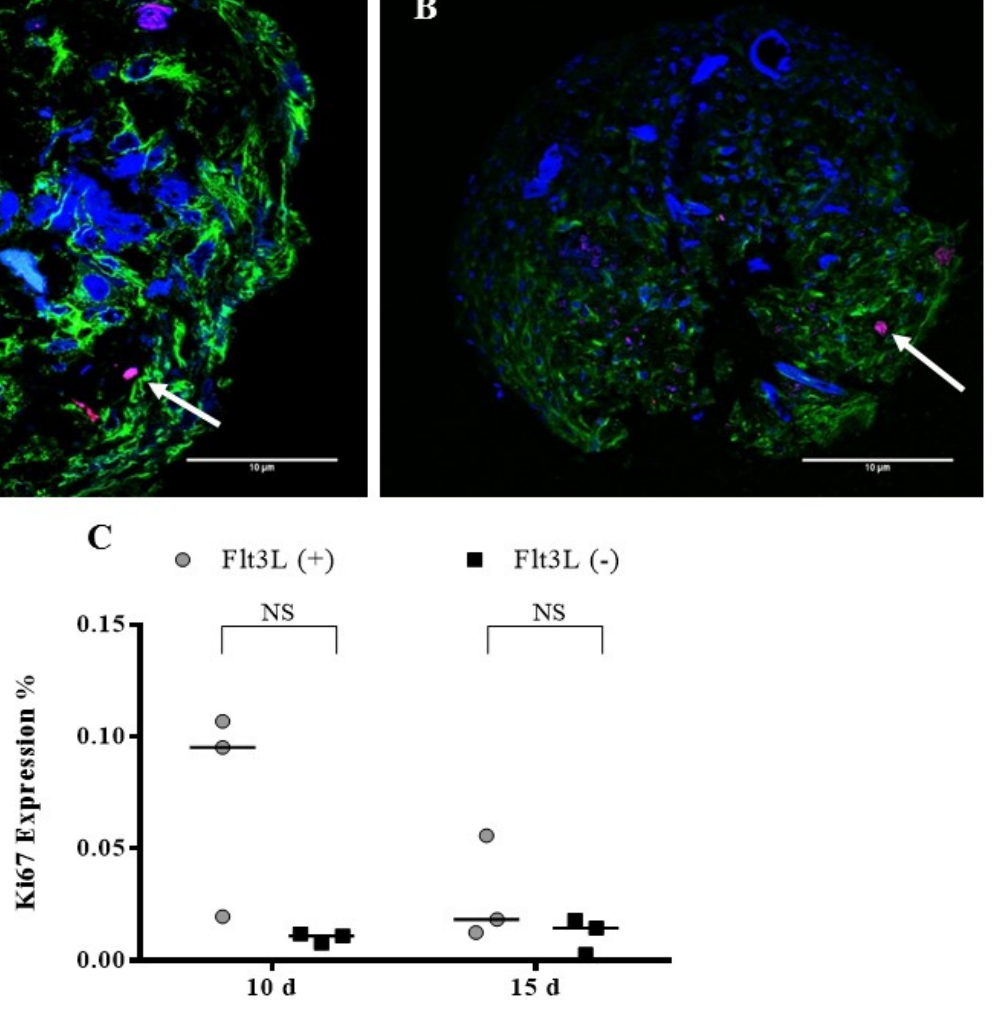

Fig. 6. Ki67 expression. OMS were cultivated with (A) Flt3L (+) and (B) without the cytokine Flt3L (cells expressing Ki67 antigen are indicated with the arrows). Cuts of the spheres were imaged with laser scanning confocal microscope FV1000 (Olympus) using an UPLSAPO $20 \times, 1.35$ NA oil immersion objective. A 405 $\mathrm{nm}$ diode laser was used for DAPI (nuclei), $488 \mathrm{~nm}$ argon laser line for Alexa-Fluor $488 \mathrm{~nm}$ ( $\alpha$-tubulin) and Argon $515 \mathrm{~nm}$ laser for alexa-fluor $514 \mathrm{~nm}$ (Ki67). Data analysis was performed with the FlowView and Image) software (green: $\alpha$-tubulin, blue: DAPI, magenta: Ki67) (Scale bar: $10 \mu \mathrm{m})$. (C) Percentage of Ki67 expression after 10 and $15 \mathrm{~d}$. 
A

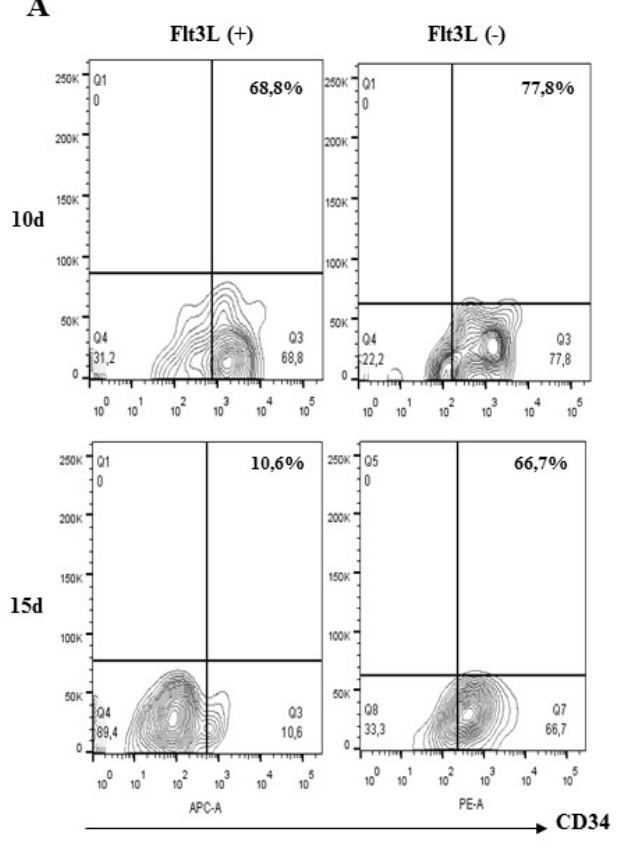

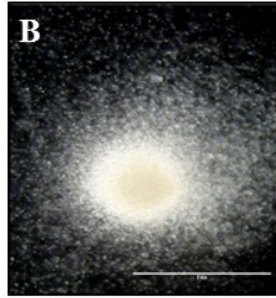
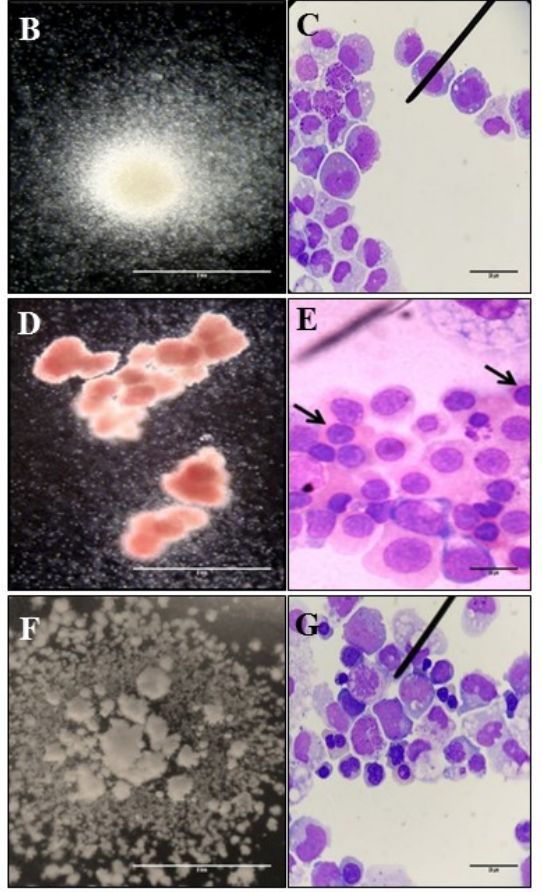

H

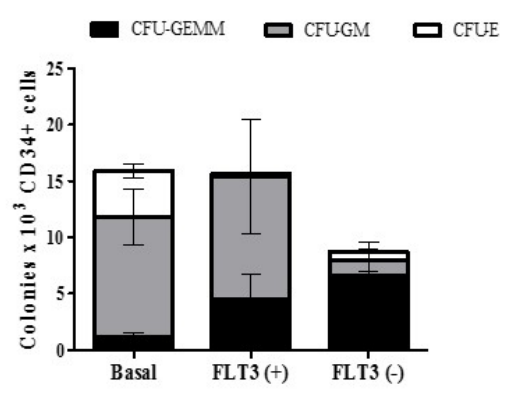

I

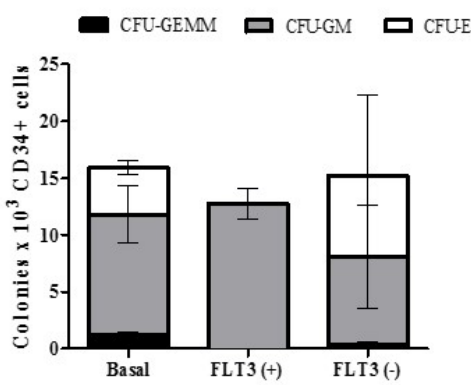

Fig. 7. Multipotent capacity of HSC after cultivation in OMS. (A) Dot plot representative of the recovery percentages of CD34+ cells after the disintegration of the multicellular spheres. The ability of HSC to generate (B) CFU-GM (colony forming unit - granulocyte/monocyte progenitor. $\times 10$, inverted microscope. Scale bar: $5 \mathrm{~mm}$ ) (C) Immature granulocytic and monocytic cells: promyelocytes, myelocytes, promonocytes and bands cells $(\times 100$, hematoxylin \& eosin. Scale bar: $20 \mu \mathrm{m})$, (D) CFU-E (colony forming unit - erythroid progenitor. $\times 10$, inverted microscope. Scale bar: $5 \mathrm{~mm}$ ), (E) Immature erythroid cells: orthochromatic normoblasts are indicated ( $\times 100$, hematoxylin \& eosin. Scale bar: $20 \mu \mathrm{m}$ ), (F) CFU-GEMM (colony forming unit - granulocyte, erythrocyte, monocyte, megakaryocyte. $\times 10$, inverted microscope. Scale bar: $5 \mathrm{~mm})$ and $(\mathrm{G})$ Immature cells of myeloid lineage: myeloblasts, promyelocytes, normoblasts, promonocytes $(\times 100$, hematoxylin \& eosin. Scale bar: $20 \mu \mathrm{m})$ was evaluated after $10 \mathrm{~d}(\mathrm{H})$ and $15 \mathrm{~d}(\mathrm{I})(\mathrm{n}=1)(\mathrm{p}>0.05)$.

spheroids in non-adherent culture conditions (36, 37), but the generation of OMS from human normal cells with a profile of adhesion molecules different from tumor cells to mimic the microenvironment of a "semi-solid" tissue, such as bone marrow, is a different challenge. The use of the magnetic levitation system allowed us to generate a sphere with adherent cellular populations, such as MSC and Ec, and non-adherent cells, such as HSC, which interact in an OMS that retains its viability for 15 days (Fig. 5C-F). Besides that the magnetic levitation system allowed us to develop a multicellular sphere from normal cell populations with different profiles of adhesion molecules, previous studies have shown that this system does not require the use of exogenous proteins or synthetic scaffolds that modify the cellular physiology but promotes the production of proteins of the extracellular matrix recreating cellular microenvironments similar to those that exist in vivo $(38,39)$. Since our interest was to develop a 3D culture system that mimics the conditions of the HSC microenvironment, we selected this system for the advantages described above.
In relation to the histological evaluation of the spheres, we showed that they have an organization defined by a cell population that is organized around the perimeter of the structure (Fig. 3G) and other cell populations distributed in the center of the structure that do not have pyknotic nuclei associated with a necrotic center, as described in spheres obtained with tumor cells (40); this is an important result because it indirectly demonstrates the maintenance of cell viability within the 3D structure and the non-toxicity of the nanoparticles used in the magnetic levitation system. We also evaluated the global expression of vimentin in OMS. This is a protein that is used in histological studies to demonstrate the maintenance of cell integrity because it has been shown that its decrease is related to apoptosis $(41,42)$; therefore, in our study we used the detection of vimentin as an indicator of the quality of the structure rather than a protein associated with mesenchymal cells where it is abundantly expressed. We observed that there is a correlation between the morphological findings observed inside the sphere (Fig. 3G) without apoptotic cells and a high expression of vimentin in 
the structures (Fig. 5K, L) demonstrated the formation of spheres with histological quality.

Our goal was to develop an in vitro system that would allow us to imitate the microenvironment of human HSC (vascular-stromal niche) without the exogenous use of proteins or scaffolds. It has been demonstrated that the exogenous addition only of the FLt3-L can synergize with the SCF (Stem Cell Factor) and TPO (thrombopoietin) secreted by the mesenchymal cells, stimulating the proliferation and maintenance of the HSC (27); therefore, to evaluate whether the obtained OMS can autonomously maintain the $\mathrm{CD} 34+$ cells or require the addition of exogenous hematopoietic cytokines, we use the Flt3L cytokine. Additionally, we evaluated the effect of Flt3L on the formation of the structure (percentage of aggregation), viability and expression of Ki67.

We did not observe differences in the percentage of aggregation (Fig. 4G) and viability (Fig. 5C-F) between the OMS cultivated with Flt3L and without the cytokine. We observed a greater tendency in the expression of Ki67 in the sphere with Flt3L (Fig. 6C) although the difference was not significant. After isolating the cultured HSC from OMS (10 and 15 days), we could determine that our structures maintain viable and multipotent HSC without the exogenous addition of cytokine. The multipotency test showed that from HSC cultivated in the OMS with Flt3-L, a greater number of committed progenitors colonies (CFU-GM and CFU-E) is obtained, while cultivating HSC in the cytokine-free makes them capable of generating a greater number of myeloid progenitors (CFU-GEMM) (Fig. 7). This shows that the OMS cultivated without the addition of exogenous cytokines is autonomous in the maintenance of HSC and that the survival and functionality of these cells depend only on their interaction with MSC and Ec and the soluble factors secreted by these cells as they occur in vivo. This is particularly interesting in HSCs isolated from umbilical cord blood because this source has a very low number of HSCs compared to BM or peripheral blood mobilized (25), and although no significant increase in the number of HSCs is observed through the OMS, an important increase in the number of myeloid progenitors after culture was observed (Fig. 7, basal vs. Flt3L-), which could contribute to the efficiency in the repopulation capacity of this cellular population in adult recipient patients despite the number of available cells.

\section{Conclusions}

In summary, our results demonstrate the development of an OMS from normal human cells as a model for the study of the microenvironment of human HSC. This type of development will allow for the generation of more complex spheroids that imitate the microenvironment of human stem cells and the proposal of new protocols in regenerative medicine.

\section{Acknowledgments}

The authors would like to thank to staff members from the Department of Gynecology and Obstetrics, Department of Orthopedics and Traumatology and Department of Pathology in the Hospital Universitario San Ignacio (Bogotá, Colombia). We also wish to thank all the patients who voluntarily donated the tissues used in this study. Special thanks to COLCIENCIAS and Pontificia Universidad Javeriana for funding this work.

\section{Potential Conflict of Interest}

The authors declare that there is no conflict of interest.

\section{References}

1. Schofield R. The relationship between the spleen colony-forming cell and the haemopoietic stem cell. Blood Cells 1978;4:7-25

2. Morrison SJ, Scadden DT. The bone marrow niche for haematopoietic stem cells. Nature 2014;505:327-334

3. Calvi LM, Link DC. The hematopoietic stem cell niche in homeostasis and disease. Blood 2015;126:2443-2451

4. Asada N, Kunisaki Y, Pierce H, Wang Z, Fernandez NF, Birbrair A, Ma'ayan A, Frenette PS. Differential cytokine contributions of perivascular haematopoietic stem cell niches. Nat Cell Biol 2017;19:214-223

5. Mangialardi G, Cordaro A, Madeddu P. The bone marrow pericyte: an orchestrator of vascular niche. Regen Med 2016;11:883-895

6. Ding L, Morrison SJ. Haematopoietic stem cells and early lymphoid progenitors occupy distinct bone marrow niches. Nature 2013;495:231-235

7. Kobayashi H, Butler JM, O'Donnell R, Kobayashi M, Ding BS, Bonner B, Chiu VK, Nolan DJ, Shido K, Benjamin L, Rafii S. Angiocrine factors from Akt-activated endothelial cells balance self-renewal and differentiation of haematopoietic stem cells. Nat Cell Biol 2010;12:1046-1056

8. Winkler IG, Barbier V, Nowlan B, Jacobsen RN, Forristal CE, Patton JT, Magnani JL, Lévesque JP. Vascular niche E-selectin regulates hematopoietic stem cell dormancy, self renewal and chemoresistance. Nat Med 2012;18:1651-1657

9. Chute JP, Muramoto GG, Dressman HK, Wolfe G, Chao NJ, Lin S. Molecular profile and partial functional analysis of novel endothelial cell-derived growth factors that regulate hematopoiesis. Stem Cells 2006;24:1315-1327

10. Li W, Johnson SA, Shelley WC, Yoder MC. Hematopoietic stem cell repopulating ability can be maintained in vitro 
by some primary endothelial cells. Exp Hematol 2004;32: 1226-1237

11. Ding L, Saunders TL, Enikolopov G, Morrison SJ. Endothelial and perivascular cells maintain haematopoietic stem cells. Nature 2012;481:457-462

12. Greenbaum A, Hsu YM, Day RB, Schuettpelz LG, Christopher MJ, Borgerding JN, Nagasawa T, Link DC. CXCL12 in early mesenchymal progenitors is required for haematopoietic stem-cell maintenance. Nature 2013;495: 227-230

13. Kunisaki Y, Bruns I, Scheiermann C, Ahmed J, Pinho S, Zhang D, Mizoguchi T, Wei Q, Lucas D, Ito K, Mar JC, Bergman A, Frenette PS. Arteriolar niches maintain haematopoietic stem cell quiescence. Nature 2013;502:637-643

14. Rodríguez-Pardo VM, Aristizabal JA, Jaimes D, Quijano SM, de los Reyes I, Herrera MV, Solano J, Vernot JP. Mesenchymal stem cells promote leukaemic cells aberrant phenotype from B-cell acute lymphoblastic leukaemia. Hematol Oncol Stem Cell Ther 2013;6:89-100

15. Rodríguez-Pardo VM, Vernot JP. Mesenchymal stem cells promote a primitive phenotype $\mathrm{CD} 34+\mathrm{c}-\mathrm{kit}+$ in human cord blood-derived hematopoietic stem cells during ex vivo expansion. Cell Mol Biol Lett 2013;18:11-33

16. Boieri $M$, Shah $P$, Dressel $R$, Inngjerdingen $M$. The role of animal models in the study of hematopoietic stem cell transplantation and GvHD: a historical overview. Front Immunol 2016;7:333

17. Méndez-Ferrer S, Michurina TV, Ferraro F, Mazloom AR, Macarthur BD, Lira SA, Scadden DT, Ma'ayan A, Enikolopov GN, Frenette PS. Mesenchymal and haematopoietic stem cells form a unique bone marrow niche. Nature 2010;466:829-834

18. Pinho S, Marchand T, Yang E, Wei Q, Nerlov C, Frenette PS. Lineage-biased hematopoietic stem cells are regulated by distinct niches. Dev Cell 2018;44:634-641.e4

19. Reichert D, Friedrichs J, Ritter S, Käubler T, Werner C, Bornhäuser M, Corbeil D. Phenotypic, morphological and adhesive differences of human hematopoietic progenitor cells cultured on murine versus human mesenchymal stromal cells. Sci Rep 2015;5:15680

20. van Pel M, Fibbe WE, Schepers K. The human and murine hematopoietic stem cell niches: are they comparable? Ann N Y Acad Sci 2016;1370:55-64

21. Simian M, Bissell MJ. Organoids: a historical perspective of thinking in three dimensions. J Cell Biol 2017;216:31-40

22. Jaganathan H, Gage J, Leonard F, Srinivasan S, Souza GR, Dave B, Godin B. Three-dimensional in vitro co-culture model of breast tumor using magnetic levitation. Sci Rep 2014;4:6468

23. Kelm JM, Timmins NE, Brown CJ, Fussenegger M, Nielsen LK. Method for generation of homogeneous multicellular tumor spheroids applicable to a wide variety of cell types. Biotechnol Bioeng 2003;83:173-180

24. Martin I, Dozin B, Quarto R, Cancedda R, Beltrame F. Computer-based technique for cell aggregation analysis and cell aggregation in in vitro chondrogenesis. Cytometry
1997;28:141-146

25. Hordyjewska A, Popiołek $€$, Horecka A. Characteristics of hematopoietic stem cells of umbilical cord blood. Cytotechnology 2015;67:387-396

26. Bari S, Seah KK, Poon Z, Cheung AM, Fan X, Ong SY, Li S, Koh LP, Hwang WY. Expansion and homing of umbilical cord blood hematopoietic stem and progenitor cells for clinical transplantation. Biol Blood Marrow Transplant 2015;21:1008-1019

27. Oubari F, Amirizade N, Mohammadpour H, Nakhlestani $M$, Zarif MN. The important role of FLT3-L in ex vivo expansion of hematopoietic stem cells following co-culture with mesenchymal stem cells. Cell J 2015;17:201-210

28. Dominici M, Le Blanc K, Mueller I, Slaper-Cortenbach I, Marini F, Krause D, Deans R, Keating A, Prockop Dj, Horwitz E. Minimal criteria for defining multipotent mesenchymal stromal cells. The International Society for Cellular Therapy position statement. Cytotherapy 2006;8: 315-317

29. Moscona A, Moscona $\mathrm{H}$. The dissociation and aggregation of cells from organ rudiments of the early chick embryo. J Anat 1952;86:287-301

30. Abu-Absi SF, Friend JR, Hansen LK, Hu WS. Structural polarity and functional bile canaliculi in rat hepatocyte spheroids. Exp Cell Res 2002;274:56-67

31. Mironov V, Visconti RP, Kasyanov V, Forgacs G, Drake CJ, Markwald RR. Organ printing: tissue spheroids as building blocks. Biomaterials 2009;30:2164-2174

32. Robinson EE, Foty RA, Corbett SA. Fibronectin matrix assembly regulates alpha5betal-mediated cell cohesion. Mol Biol Cell 2004;15:973-981

33. Shimazui T, Schalken JA, Kawai K, Kawamoto R, van Bockhoven A, Oosterwijk E, Akaza H. Role of complex cadherins in cell-cell adhesion evaluated by spheroid formation in renal cell carcinoma cell lines. Oncol Rep 2004; 11:357-360

34. Lee BH, Kim MH, Lee JH, Seliktar D, Cho NJ, Tan LP. Modulation of Huh7.5 spheroid formation and functionality using modified PEG-based hydrogels of different stiffness. PLoS One 2015;10:e0118123

35. Lin RZ, Chou LF, Chien CC, Chang HY. Dynamic analysis of hepatoma spheroid formation: roles of E-cadherin and betal-integrin. Cell Tissue Res 2006;324:411-422

36. Weiswald LB, Bellet D, Dangles-Marie V. Spherical cancer models in tumor biology. Neoplasia 2015;17:1-15

37. Morata-Tarifa C, Jiménez G, García MA, Entrena JM, Griñán-Lisón C, Aguilera $M$, Picon-Ruiz M, Marchal JA. Low adherent cancer cell subpopulations are enriched in tumorigenic and metastatic epithelial-to-mesenchymal transition-induced cancer stem-like cells. Sci Rep 2016;6: 18772

38. Haisler WL, Timm DM, Gage JA, Tseng H, Killian TC, Souza GR. Three-dimensional cell culturing by magnetic levitation. Nat Protoc 2013;8:1940-1949

39. Souza GR, Molina JR, Raphael RM, Ozawa MG, Stark DJ, Levin CS, Bronk LF, Ananta JS, Mandelin J, Georgescu 
MM, Bankson JA, Gelovani JG, Killian TC, Arap W, Pasqualini R. Three-dimensional tissue culture based on magnetic cell levitation. Nat Nanotechnol 2010;5:291-296

40. Lin RZ, Chang HY. Recent advances in three-dimensional multicellular spheroid culture for biomedical research. Biotechnol J 2008;3:1172-1184

41. Goldman RD, Khuon S, Chou YH, Opal P, Steinert PM.
The function of intermediate filaments in cell shape and cytoskeletal integrity. J Cell Biol 1996;134:971-983

42. Morishima N. Changes in nuclear morphology during apoptosis correlate with vimentin cleavage by different caspases located either upstream or downstream of $\mathrm{Bcl}-2$ action. Genes Cells 1999;4:401-414 